\title{
Stress myocardial perfusion gated-SPECT imaging in advanced chronic kidney disease
}

\author{
Pierre-Yves Marie, MD, PhD, ${ }^{\mathrm{a}, \mathrm{b}}$ and Patrick Rossignol, $M D, \mathrm{PhD}^{\mathrm{b}, \mathrm{c}}$ \\ a CHRU-Nancy, Université de Lorraine, Nuclear Medicine \& Nancyclotep Experimental Platform, \\ Nancy, France \\ b Université de Lorraine, INSERM, UMR-1116, Nancy, France \\ c Université de Lorraine, CHRU-Nancy, INSERM 1433 Centre D'Investigation Clinique \& FCRIN \\ INI-CRCT (Cardiovascular and Renal Clinical Trialists), Nancy, France
}

Received May 20, 2018; accepted May 21, 2018

doi: $10.1007 / \mathrm{s} 12350-018-1412-7$

\section{See related article, pp. 1962-1970}

Cardiovascular death is a leading cause of death in patients with chronic kidney disease (CKD), while the risk of cardiovascular mortality, adjusted for traditional cardiovascular risk factors and albuminuria, is linearly and negatively correlated with renal function. ${ }^{1}$ In particular, cardiovascular mortality in CKD patients who are on dialysis is 10 to 20 times higher than in the general population. ${ }^{2,3}$

This increased cardiovascular risk primarily concerns coronary artery disease $(\mathrm{CAD}),{ }^{4}$ with $\mathrm{CKD}$ patients exhibiting high prevalences of traditional cardiovascular risk factors (advanced age, hypertension, diabetes, dyslipidemia) as well as certain uremia-specific risk factors. ${ }^{5}$

It should also be borne in mind that the atherosclerotic process in CKD patients can remain highly active even after renal transplantation, with hypertension, hyperlipidemia, and hyperglycemia being further facilitated by immunosuppressant therapy (corticosteroids, calcineurin inhibitors). Indeed, the mortality of CKD patients remains of cardiovascular origin in $35 \%$ to $50 \%$ of cases after transplantation. ${ }^{4}$

See related article, https://doi.org/10.1007/s12350-018-1411-8.

Reprint requests: Pierre-Yves Marie, MD, PhD, CHRU-Nancy, Université de Lorraine, Nuclear Medicine \& Nancyclotep Experimental Platform, Nancy, France; py.marie@chru-nancy.fr

J Nucl Cardiol 2019;26:1971-3.

$1071-3581 / \$ 34.00$

Copyright (C) 2018 American Society of Nuclear Cardiology.
Of importance is the lack of demonstration to date of the efficacy of myocardial revascularization procedures in advanced CKD patients. Fortunately, the ongoing ISCHEMIA CKD trial aims to determine the best management strategy for patients with advanced CKD and at least moderate ischemia, and randomized to either (1) an invasive strategy with cardiac catheterization followed, if suitable, by revascularization or (2) a conservative strategy with catheterization and revascularization reserved in the event of failure of optimal medical therapy (https://clinicaltrials.gov/ct2/show/ NCT01985360).

The medical management of CAD may need to be adapted to the CKD stage, as well as be initiated as early as possible, although the latter is contingent on our ability to detect early stages of CAD. Unfortunately, this diagnosis may be difficult, partly because myocardial ischemia is frequently silent in CKD patients, owing to diabetic and/or uremic neuropathies, and because a maximal exercise test is often difficult to obtain, especially in the more fragile patients with end-stage CKD. In addition, CT angiography is frequently hampered by high rates of coronary calcification and carries a risk of further worsening of renal function through contrastinduced nephropathy.

In this particular setting, myocardial perfusion SPECT imaging (MPI) likely offers the advantages (1) of being relatively simple to perform and to analyze, including for the growing number of patients for whom MRI or echocardiography is contraindicated or unreliable (pacemaker and certain metallic implants, atrial fibrillation and other rhythm disturbances, poor acoustic window, etc.) and (2) of providing a wide variety of stress methods, allowing this technique to be adapted to each patient, even for the more fragile individuals. ${ }^{6}$ In addition, MPI has taken advantage of major technical 
advances in recent years, and can now be performed with low radiation exposure $(\leq 5 \mathrm{mSv}){ }^{7}$ allowing MPI to be repeated much more frequently throughout the patients' lifetime.

It has long been shown that an abnormal stress MPI is associated with a clear increase in the risk of cardiac ischemic events in CKD patients, although equivalent results have also been known to be achieved with dobutamine stress echocardiography. ${ }^{8}$ However, the dobutamine test may likely have the disadvantage of a higher risk of adverse events, compared with all other stress techniques. ${ }^{9}$

Conversely, a normal stress MPI is associated with a fairly good outcome in CKD patients. ${ }^{8,10}$ Nevertheless, this outcome is significantly worse when only considering patients with very poor renal function and those with certain additional risk factors, such as low plasma hemoglobin and/or high C-reactive protein levels. ${ }^{10}$ In particular, in CKD patients with normal stress MPI and a normal left ventricular (LV) function at gated-SPECT, the major cardiovascular event rate was recently found to be twice as high for those with an estimated glomerular filtration rate (eGFR) $<15 \mathrm{~mL} / \mathrm{min}$ than for those with an eGFR $\geq 30 \mathrm{~mL} / \mathrm{min} .{ }^{10}$ This can presumably be explained by the accelerated rates by which coronary atherosclerosis may develop in the more severe stages of CKD. ${ }^{5}$ This consideration is further strengthened by the recent observation of a particularly high rate of new-onset ischemia in patients awaiting renal transplantation and subjected to serial stress MPI. ${ }^{11}$

The key question at this juncture is how to do better and how to enhance the risk assessment and medical management of CKD patients in diagnostic strategies involving stress MPI?

A first possibility is indubitably that of a better integration of MPI data with the results of other investigative tests such as certain very simple blood biomarkers providing additional and independent prognostic information, as stated above. For instance, a high C-reactive protein blood level $(>0.3 \mathrm{mg} / \mathrm{dL})$ was recently shown to be associated with a marked increase in cardiovascular risk for CKD patients with normal stress MPI and normal LV function at gated-SPECT. ${ }^{10}$ By contrast, it should be pointed out that the coronary artery calcium score, such as that provided with current hybrid SPECT/CT systems, does not offer incremental prognostic information relative to that from stress MPI in patients with advanced $\mathrm{CKD},{ }^{12}$ contrary to that previously documented in more general study populations.

Information on LV systolic function provided by gated-SPECT may also be useful in this setting, keeping in mind that this assessment may now be obtained with very low radiation doses on recent CZT cameras. ${ }^{13}$ In particular, in a still ongoing multicenter prospective trial, end-systolic volume, determined with gatedSPECT, was found to provide strong predictive information on the risk of cardiac death, in addition to information provided by the extent of stress perfusion abnormalities. ${ }^{14}$

In addition to the above, very promising gatedSPECT results have been provided to date by phase parameters reflecting mechanical $L V$ dyssynchrony with strong relationships with the prognosis of CKD patients, independently of myocardial perfusion and LV ejection fraction. ${ }^{15-17}$

LV dyssynchrony parameters, extracted from the phase histogram of gated-SPECT studies (i.e., the bandwidth and standard deviation of the phase), have already been shown to aid in determining the prognosis of heart failure patients, as well as the likelihood of effective response to resynchronization therapy. ${ }^{18}$ However, LV dyssynchrony is caused not only by electrical remodeling and/or myocardial tissue damage, but also by structural remodeling and abnormal volume loading of the LV, all these disorders being currently associated with advanced CKD.

LV dyssynchrony has also been shown to be generally reduced when LV volume and preload are decreased by the initiation of hemodialysis treatment. ${ }^{19}$ However, in an original research study conducted by Angelidis et al. and presented in this issue of the Journal of Nuclear Cardiology, ${ }^{20} \mathrm{LV}$ dyssynchrony parameters provided by gated-SPECT were found unchanged by renal transplantation, whereas a definite improvement was documented for LV ejection fraction and volume at the same time points. This observation could be explained by an irreversible or poorly reversible LV remodeling and fibrosis, thus strengthening the consideration that there is a need to further assess specific therapies in this setting. The ongoing ALdosterone Antagonist Chronic HEModialysis Interventional Survival Trial (ALCHEMIST, NCT01848639) is currently assessing the effects of a mineralocorticoid receptor antagonist displaying antifibrotic properties on the prognosis of CKD patients treated by dialysis.

Finally, it is likely that stress MPI performed with the more recent high-sensitivity cameras and integrating several gated-SPECT parameters (i.e., ejection fraction and volume, as well as phase parameters) may ultimately provide a comprehensive analysis of a number of factors involved in the prognosis of CKD patients, and thereby could putatively reach the status of first line technique, in complement to echocardiography, for the serial cardiac monitoring of CKD patients. 


\section{Acknowledgements}

Pierre-Yves Marie and Patrick Rossignol are supported by the French National Research Agency (ANR) as part of the second 'Investissements d'Avenir' programme (reference: ANR-15-RHU-0004).

\section{Disclosure}

The authors declare that they have no conflict of interest.

\section{References}

1. Gansevoort RT, Correa-Rotter R, Hemmelgarn BR, Jafar TH, Heerspink HJ, Mann JF, et al. Chronic kidney disease and cardiovascular risk: Epidemiology, mechanisms, and prevention. Lancet 2013;382:339-52.

2. Ortiz A, Covic A, Fliser D, Fouque D, Goldsmith D, Kanbay M, et al. Epidemiology, contributors to, and clinical trials of mortality risk in chronic kidney failure. Lancet 2014;383:1831-43.

3. Rossignol P, Pitt B, Thompson A, Zannad F. Roadmap for cardiovascular prevention trials in chronic kidney disease. Lancet 2016;388(10055):1964-6.

4. Cai Q, Mukku VK, Ahmad M. Coronary artery disease in patients with chronic kidney disease: A clinical update. Curr Cardiol Rev 2013;9(4):331-9.

5. Vanholder R, Fouque D, Glorieux G, Heine GH, Kanbay M, Mallamaci $\mathrm{F}$, et al. Clinical management of the uraemic syndrome in chronic kidney disease. Lancet Diabetes Endocrinol 2016;4(4):360-73.

6. Cho SG, Jabin Z, Bom HH. Safer stress tests for myocardial perfusion imaging. J Nucl Cardiol 2018. https://doi.org/10.1007/ s12350-017-1083-9.

7. Imbert L, Marie PY. CZT cameras: A technological jump for myocardial perfusion SPECT. J Nucl Cardiol 2016;23(4):894-6.

8. Rabbat CG, Treleaven DJ, Russell JD, Ludwin D, Cook DJ. Prognostic value of myocardial perfusion studies in patients with end-stage renal disease assessed for kidney or kidney-pancreas transplantation: A meta-analysis. J Am Soc Nephrol 2003;14(2):431-9.

9. Dilsizian V, Gewirtz H, Paivanas N, Kitsiou AN, Hage FG, Crone $\mathrm{NE}$, et al. Serious and potentially life threatening complications of cardiac stress testing: Physiological mechanisms and management strategies. J Nucl Cardiol 2015;22:1198-213.

10. Matsuo S, Nakajima K, Takeishi Y, Nishimura T. Prognostic value of normal stress myocardial perfusion imaging and ventricular function in Japanese patients with chronic kidney disease: A study based on the J-ACCESS-3 database. Eur J Nucl Med Mol Imaging 2018;45:1101-7.

11. Moody WE, Lin ELS, Thomson LE, Berman DS, Edwards NC, Holloway B, et al. Results of serial myocardial perfusion imaging in end-stage renal disease. Am J Cardiol 2018;121(5):661-7.

12. Moody WE, Lin EL, Stoodley M, McNulty D, Thomson LE, Berman DS, et al. Prognostic utility of calcium scoring as an adjunct to stress myocardial perfusion scintigraphy in end-stage renal disease. Am J Cardiol 2016;117(9):1387-96.

13. Claudin M, Imbert L, Djaballah W, Veran N, Poussier S, Roch V, et al. Routine evaluation of left ventricular function using CZTSPECT, with low injected activities and limited recording times. J Nucl Cardiol 2018;25(1):249-56.

14. Kasama S, Toyama T, Sato M, Sano H, Ueda T, Sasaki T, et al. Prognostic value of myocardial perfusion single photon emission computed tomography for major adverse cardiac cerebrovascular and renal events in patients with chronic kidney disease: Results from first year of follow-up of the Gunma-CKD SPECT multicenter study. Eur J Nucl Med Mol Imaging 2016;43(2):302-11.

15. AlJaroudi W, Aggarwal H, Venkataraman R, Heo J, Iskandrian AE, Hage FG. Impact of left ventricular dyssynchrony by phase analysis on cardiovascular outcomes in patients with end-stage renal disease. J Nucl Cardiol 2010;17:1058-64.

16. Aggarwal H, AlJaroudi WA, Mehta S, Mannon R, Heo J, Iskandrian AE, et al. The prognostic value of left ventricular mechanical dyssynchrony using gated myocardial perfusion imaging in patients with end-stage renal disease. J Nucl Cardiol 2014;21:73946.

17. Mori H, Isobe S, Suzuki S, Unno K, Morimoto R, Kano N, et al. Prognostic value of left ventricular dyssynchrony evaluated by gated myocardial perfusion imaging in patients with chronic kidney disease and normal perfusion defect scores. J Nucl Cardiol 2018. https://doi.org/10.1007/s12350-017-0889-9.

18. Naya M, Manabe O, Koyanagawa K, Tamaki N. The role of nuclear medicine in assessments of cardiac dyssynchrony. J Nucl Cardiol 2018. https://doi.org/10.1007/s12350-017-1072-z.

19. Takahashi N, Sato N, Ishikawa M, Kikuchi A, Hanaoka D, Ishihara $\mathrm{S}$, et al. Long-term hemodialysis corrects left ventricular dyssynchrony in end-stage renal disease: A study with gated technetium-99m sestamibi myocardial perfusion single-photon emission computed tomography. J Nippon Med Sch 2015;82(2):76-83.

20. Crosland W, Aggarwal H, Farag A, Mehta S, Mannon RB, Heo J, et al. The effect of renal transplantation on left ventricular function, electrocardiography, and mechanical synchrony by gated myocardial perfusion imaging. J Nucl Cardiol 2018. 